\title{
Efficacy of Salvage Interferential Electrical Stimulation Therapy in Patients With Medication-Refractory Enuresis: A Pilot Study
}

\author{
Hahn-Ey Lee, Kwanjin Park \\ Department of Pediatric Urology, Seoul National University Children's Hospital, Seoul, Korea
}

\begin{abstract}
Purpose: In pediatric patients with enuresis, the protocol for salvage therapy in patients in whom first-line therapy was not successful has not yet been established. Interferential electrical stimulation (IF-ES) therapy is advantageous because it is noninvasive and shows high compliance. We aimed to investigate the efficacy and safety of IF-ES therapy on pediatric enuresis in a pilot study. Methods: We investigated 10 patients who underwent IF-ES therapy between August 2012 and March 2013 at our clinic. Patients with a history of previous treatment with desmopressin and anticholinergic agents for at least 3 months and those in whom alarm treatment previously failed or was refused by parents were eligible. Electrical current was given starting at approximately $20 \mathrm{~mA}$ and was increased until the patient complained of discomfort. Treatment was performed once a week, 20 minutes per treatment, 6 times per cycle. After each cycle, an interview was performed and voiding diaries were filled. The physician in charge evaluated improvement according to the International Children's Continence Society criteria.

Results: A final analysis was performed in 10 patients ( 5 male and 5 female patients) in whom therapy for nocturnal enuresis had failed. Eight patients had nonmonosymptomatic enuresis and 2 had monosymptomatic enuresis. The mean age of the patients was $8.5 \pm 2.4$ years, and the mean number of treatments was $10.6 \pm 3.6$ times. A full response was observed in 1 patient (10\%); a good response, in 1 patient (10\%); a partial response, in 7 patients (70\%); and no response, in 1 patient (10\%).

Conclusions: Our study demonstrated that IF-ES therapy can be a promising treatment for the future, is safe, and can benefit from appropriate clinical trials in carefully selected groups. IF-ES therapy is expected to be a safe and effective treatment modality for children with enuresis.
\end{abstract}

Keywords: Electric stimulation; Enuresis; Salvage therapy

\section{INTRODUCTION}

Enuresis is defined by the International Children's Continence Society (ICCS) as loss of discrete amounts of urine during sleep in children older than 5 years [1]. Enuresis in children is generally benign but can cause emotional stress to children and their parents [2]. The incidence of enuresis in Korean children has not yet been reported; however, Lee et al. [3] reported that the incidence of enuresis in the Pusan province of Korea was similar to that in European children (12.8\%). In Seoul National University Children's Hospital, we often encounter children with enuresis, and some do not show symptom improvement with conventional treatment.
Nocturnal enuresis (NE) can be classified as monosymptomatic enuresis (MNE) and nonmonosymptomatic enuresis (NMNE) according to the ICCS $[4,5]$. MNE is defined as enuresis in children without any other lower urinary tract symptoms (LUTS), and NMNE is defined as enuresis in children with LUTS and with a history of bladder dysfunction. The treatment for NE is identical in both MNE and NMNE, although in NMNE, underlying bowel symptoms and LUTS require treatment before treatment of NE. The treatment protocol for NE recommended by the ICCS is initially lifestyle modification, followed by alarm treatment, and pharmacotherapy that includes desmopressin, anticholinergic agents, and tricyclic antidepressants. The response related to desmopressin treatment is assessed
Corresponding author: Kwanjin Park

Department of Pediatric Urology, Seoul National University Children's Hospital, 101 Daehak-ro, Jongno-gu, Seoul 110-744, Korea

Tel: +82-2-2072-0695 / Fax: +82-2-742-4665 / E-mail: urodori@naver.com

Submitted: July 20, 2013 / Accepted after revision: September 26, 2013
This is an Open Access article distributed under the terms of the Creative Commons Attribution Non-Commercial License (http://creativecommons.org/licenses/by-nc/3.0/) which permits unrestricted non-commercial use, distribution, and reproduction in any medium, provided the original work is properly cited. 
within 1 to 2 weeks, and when a response is shown, it is administered for 3 months. Improvement or remission is then determined, and physicians decide whether desmopressin should be given daily or occasionally [4]. In patients who do not respond to such therapies, further evaluation is necessary; however, no specific guidelines have been established.

As for salvage treatment, the ICCS recommends re-evaluation and repeat pharmacotherapy for MNE; electrical stimulation (ES) is a modality recommended for patients with NMNE. ES has been widely investigated as an alternative treatment method for LUTS in children [6]. Sacral transcutaneous electrical nerve stimulation (TENS), endoanal, anogenital, posterior tibial nerve stimulation, and sacral implants have been performed. Such ES methods affect neuroplasticity and affect modulation of synapses. ES therapy recovers denervation of muscle fibers and neural reconditioning [7]. Parasacral TENS (PTENS) has been proved to be effective in the treatment of NMNE [8]. De Oliveira et al. [9] recently proved the efficacy of PTENS for $\mathrm{MNE}$ in a randomized controlled trial. Interferential ES (IF-ES) is a noninvasive modality first reported in 1987 by Emmerson et al. [10] in the treatment of detrusor instability. IF current produces a $4-\mathrm{kHz}$ carrier frequency current with a sinusoidal waveform with four surface electrodes. IF therapy offers an alternative, noninvasive method of treatment [11]. Kajbafzadeh et al. [12-14] reported that IF-ES is effective for children with myelomeningocele with incontinence, neurogenic bowel dysfunction, and detrusor overactivity.

Based on such data, we aimed to investigate whether IF-ES is effective for pediatric patients with enuresis in whom pharmacotherapy failed.

\section{MATERIALS AND METHODS}

We investigated 10 patients who underwent IF-ES at our institution between August 2012 and March 2013. These 10 children (age, 6-12 years) had either MNE or NMNE, and patients with NMNE were those with enuresis and LUTS and a history of bladder dysfunction. The inclusion criteria were as follows: no improvement despite treatment with desmopressin and anticholinergic agents for at least 3 months, no improvement with previous alarm treatment, or refusal of alarm treatment. Medical history and baseline voiding diaries were obtained. Although medication was not effective, it was maintained during IF-ES treatment therapy and was discontinued when enuresis improved.
The IF-ES was performed at an outpatient basis. A total of four surface rectangular electrodes $(2.5 \mathrm{~cm} \times 3.5 \mathrm{~cm})$ producing sinusoidal currents were attached after cleaning the skin of each site with alcohol swabs. Two of these electrodes were attached to the lower abdomen below the costal margin, and two were located on the back over the muscles between T9 and L2 as described in previous studies $[11,13]$. Electrical current was given starting at approximately $20 \mathrm{~mA}$ and was increased until the patient complained of discomfort. When the patient experienced discomfort, the $\mathrm{Hz}$ of the IF-ES was not decreased, but the electrical current strength $(\mathrm{mA})$ was decreased until a tolerable level was achieved. The IF-ES protocol of the device was set at the 4-pole vector mode central wave (Gymna Duo 200, Apsun Co., Seoul, Korea). Treatment was performed once a week, 20 minutes per treatment, 6 times per cycle. After each cycle, an interview was performed and voiding diaries were filled.

The physician in charge evaluated improvement according to the ICCS criteria. Treatment outcome, using the ICCS definitions, was defined as nonresponse (0-49\% decrease), partial response (50-89\% decrease), good response ( $90 \%$ or greater decrease), and full response (100\% decrease or less than 1 symptom occurrence monthly). Such improvement was evaluated by the number of episodes of enuresis on voiding diaries.

A descriptive analysis was performed with the independent t-test method. A Mann-Whitney U-test was performed using IBM SPSS ver. 18.0 (IBM Co., Armonk, NY, USA). A P $<0.05$ was considered to be statistically significant. This study was approved by the Institutional Review Board of Seoul National University Hospital.

\section{RESULTS}

The 10 patients who underwent IF-ES were analyzed. Eight patients had NMNE and 2 had MNE. The demographics of these 10 patients were as follows: The mean \pm standard deviation age of the patients was $8.5 \pm 2.4$ years. Five patients were male, and 5 were female. The mean number of treatment was $10.6 \pm 3.6$ times, and the strength of the electrical current was 24.4-40.4 $\mathrm{mA}$ (Table 1). Based on the decrease in number of wet nights in the previous 28 days compared with the baseline symptom frequency at the start of the training, we evaluated the response of treatment according to the ICCS criteria. Full response (100\% decrease or less than one symptom occurrence monthly) was observed in 1 patient (10\%); good response ( $90 \%$ or greater decrease), in 1 patient (10\%); partial response (50-89\% decrease), 


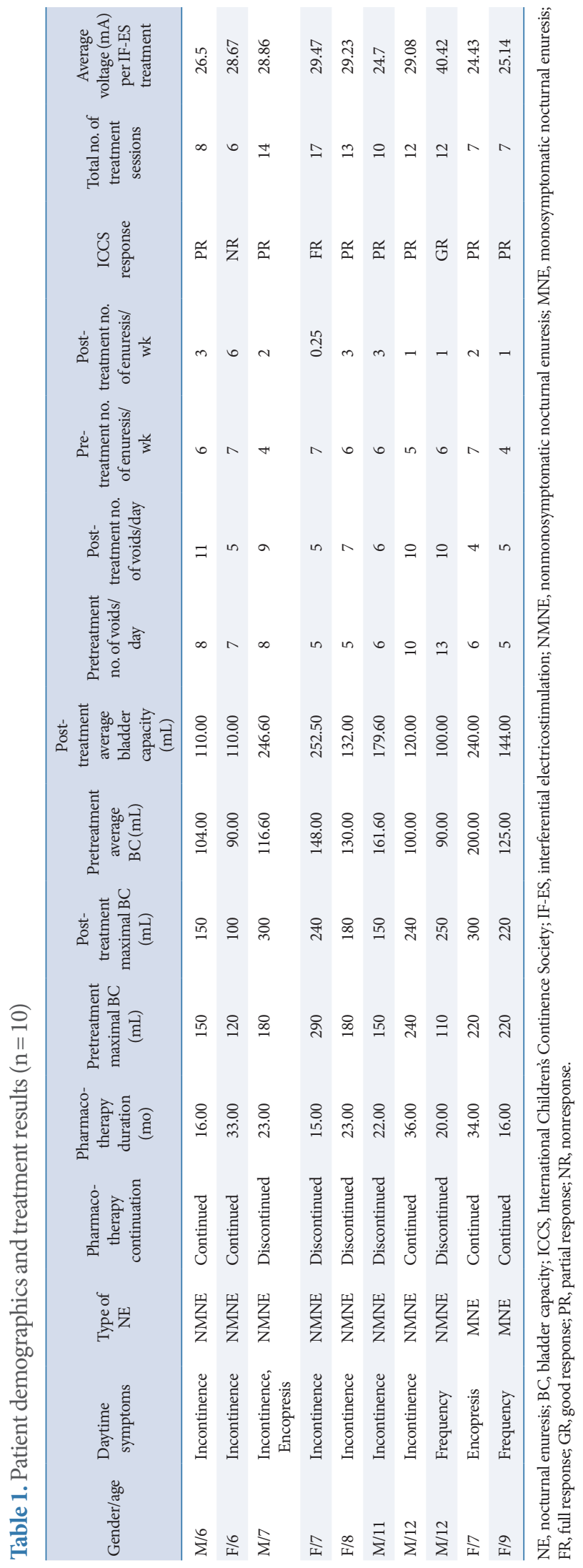

in 7 patients (70\%); and no response (0-49\% decrease), in 1 patient (10\%). Before treatment, 7 patients showed daytime urinary incontinence; 2 , daytime urinary frequency; and 2 , showed encopresis. Five patients continued pharmacotherapy during IF-ES, and 5 (50\%) stopped taking medication because symptoms improved. Three patients (30\%) showed a decrease in daytime urinary frequency, and 3 patients (30\%) showed an increase in daytime maximal bladder capacity. The mean duration of pharmacotherapy was $23.8 \pm 7.9$ months. Two patients who complained of encopresis prior to treatment reported slight improvement after cessation of treatment, although the degree of improvement was not objectively evaluated.

A comparison between NMNE and MNE groups was made (Table 2). The pretreatment and posttreatment numbers of daytime voids were higher, and the pretreatment and posttreatment number of NE episodes was lower in the MNE group, although the differences were not statistically significant.

Both pretreatment and posttreatment parameters were compared (Table 3). The mean daytime maximal bladder capacity $(\mathrm{MBC})$, which was calculated using the voiding diary maximal voided volume, showed an increase after IF-ES, although the increase was not statistically significant $(\mathrm{P}=0.210)$. The mean daytime bladder capacity also calculated by using the voiding diaries increased from $126.5 \pm 35.1 \mathrm{~mL}$ to $163.5 \pm 61.4 \mathrm{~mL}$ after treatment, and the improvement was statistically significant $(\mathrm{P}=0.026)$. The daytime number of voids showed a small decrease after treatment; however, the decrease was not statistically significant $(\mathrm{P}=0.868)$. As for the frequency of enuresis per week, the number of episodes decreased from $6.2 \pm 3.3$ times per week to $2.4 \pm 1.5$ times per week, and the decrease was statistically significant $(\mathrm{P}=0.001)$.

Factors affecting the treatment outcome were analyzed (Table 4). Age, pretreatment daytime $\mathrm{MBC}$, pretreatment daytime number of voids, pretreatment number of enuresis/week, total number of treatment sessions, and voltage $(\mathrm{mA})$ per treatment showed no statistically significant correlation.

All the patients received therapy without cessation until it was stopped by the physician. Pain was tolerable in all the patients, and no skin problems or physical discomfort were reported.

\section{DISCUSSION}

In pediatric patients with enuresis, salvage treatment after failure of first-line therapy has not yet been established. In patients with NMNE, anticholinergic agents, botulinum toxin A, cen- 
Table 2. Comparison between nonmonosymptomatic nocturnal enuresis (NMNE) and monosymptomatic nocturnal enuresis (MNE) groups

\begin{tabular}{lccc}
\hline Parameter & NMNE $(\mathrm{n}=8)$ & MNE $(\mathrm{n}=2)$ & P-value \\
Age $(\mathrm{yr})$ & $8.0 \pm 1.4$ & $8.75 \pm 2.8$ & 0.733 \\
Medication duration $(\mathrm{mo})$ & $25.0 \pm 12.7$ & $23.5 \pm 7.5$ & 0.825 \\
Pretreatment average BC (mL) & $162.5 \pm 53.0$ & $117.5 \pm 26.8$ & 0.107 \\
Post-treatment average BC (mL) & $192.0 \pm 67.9$ & $156.3 \pm 62.5$ & 0.495 \\
Pretreatment daytime MBC (mL) & $220.0 \pm 0.0$ & $177.5 \pm 60.9$ & 0.373 \\
Post-treatment daytime MBC (mL) & $260.0 \pm 56.6$ & $201.3 \pm 66.6$ & 0.289 \\
Pretreatment number of voids/day & $5.5 \pm 0.7$ & $7.8 \pm 2.7$ & 0.297 \\
Post-treatment number of voids/day & $4.5 \pm 0.7$ & $7.9 \pm 2.4$ & 0.097 \\
Pretreatment NE episodes/wk & $7.5 \pm 5.0$ & $5.9 \pm 3.2$ & 0.568 \\
Post-treatment NE episodes/wk & $1.5 \pm 0.7$ & $2.7 \pm 1.6$ & 0.372 \\
Number of IF-ES sessions & $7.0 \pm 0.0$ & $11.5 \pm 3.5$ & 0.117 \\
Average voltage (mA) per IF-ES treatment & $24.8 \pm 0.5$ & $29.6 \pm 4.7$ & 0.200 \\
\hline
\end{tabular}

Values are presented as mean \pm standard deviation.

BC, bladder capacity; MBC, maximal bladder capacity; NE, nocturnal enuresis, IF-ET; interferential electrical stimulation.

${ }^{a)}$ Analyzed by independent sample $t$-test.

Table 3. Pretreatment and post-treatment effect analysis $(n=10)$

\begin{tabular}{lccc}
\hline Parameter & Pretreatment & Post-treatment & P-value ${ }^{\mathrm{a})}$ \\
\hline Daytime maximal bladder capacity $(\mathrm{mL})$ & $183.8 \pm 56.6$ & $213.0 \pm 66.5$ & 0.210 \\
Daytime average bladder capacity $(\mathrm{mL})$ & $126.5 \pm 35.1$ & $163.5 \pm 61.4$ & $0.026^{\text {b) }}$ \\
Daytime number of voids/day & $7.3 \pm 2.6$ & $7.2 \pm 2.6$ & 0.868 \\
Number of enuresis episodes/wk & $6.2 \pm 3.3$ & $2.4 \pm 1.5$ & $0.001^{\text {b) }}$ \\
\hline
\end{tabular}

Values are presented as mean \pm standard deviation.

a) Analyzed by paired $t$-test. ${ }^{\text {b) }} \mathrm{P}<0.05$

trally active medications such as imipramine, and prostaglandin synthesis inhibitors and prostaglandin receptor antagonists can be prescribed to decrease bladder overactivity. Advanced urotherapies such as biofeedback and ES are also recommended as salvage modalities [3].

The mechanism of ES has not yet been proved; however, most studies report that afferent sacral nerve stimulation causes an interneuronal change of the spinal reflex, which induces an inhibitory stimuli in the bladder efferent nerve [15]. However, consensus has not been reached concerning the precise mechanisms, and despite multiple efforts to examine the efficacy of such salvage treatment methods using ES, no gold standard method has been established, which causes frustration to the physician and patients and family members.

In our study, the primary outcome was safety, the secondary outcome was tolerability, and the tertiary outcome was efficacy.
Although Satter [16] reported that irritation or contact dermatitis after IF-ES commonly occurs, neither condition occurred in our case. Proper electrode use and skin contact are also important to prevent electrical burns. Based on these study goals, that no adverse effects were reported was an indication that the results were satisfactory. That no patients discontinued therapy and completed all required sessions seemed to be an indicator of tolerability. That 7 patients (70\%) showed improvement in daytime urinary incontinence was a promising finding of our study. Considering that the efficacy of IF-ES on pediatric urinary incontinence has already been reported [14], such treatment benefits are attractive merits for patient and parents. An increase in daytime MBC in 3 patients (30\%) was also a promising finding.

In addition, the improvement of encopresis in $20 \%$ of the patients is an additional benefit in patients with neurogenic bowel 
Table 4. Factors affecting the outcome of interferential electrical stimulation $(\mathrm{n}=10)$

\begin{tabular}{lc}
\hline Parameter & P-value $^{\mathrm{a})}$ \\
\hline Age & 0.659 \\
Pretreatment daytime MBC & 0.750 \\
Pretreatment daytime number of voids & 0.500 \\
Pretreatment number of enuresis/wk & 0.250 \\
Total number of treatment sessions & 0.500 \\
Voltage (mA) per treatment & 1.000 \\
\hline
\end{tabular}

MBC, maximal bladder capacity.

a) Analyzed by Mann Whitney U-test.

symptoms. Queralto et al. [17] reported that interferential therapy is effective in the treatment of slow transit constipation and has less complications and is less expensive than sacral neuromodulation. Chase et al. [11] reported that when performing IF-ES in patients with urinary incontinence, an increase in bowel motility and fluid balance change occurred, suggesting the therapeutic value of both bowel and urinary symptoms after IFES.

This study has a strong advantage of being the first pilot study on the safety, tolerability, and efficacy of IF-ES in Korean pediatric patients with enuresis. Based on the results, IF-ES may be an applicable salvage modality, although the efficacy should be investigated in detail, especially in comparison with conventional methods such as TENS. For neurologically normal patients who do not response to pharmacotherapy for behavior therapy, TENS has been reported to be effective [18], and we expect that IF-ES will also be effective but with fewer adverse effects.

Despite such positive results, this study has some limitations. First, it was a short-term study, and second, because it was a pilot study, the sample size was relatively small. Due to the limited number of patients, a more detailed analysis of correlation among parameters was not possible, and a comparison between NMNE and MNE groups also had limitations. In addition, in patients with NMNE, underlying bowel symptoms and LUTS were not treated or evaluated with objective parameters, which should be supplemented in future studies. Another limitation was that considering the correlation with risk of skin burns, body mass index evaluation might prove to be valuable in future studies $[19,20]$. Another potential limitation, which is not unique to our study, is the possibility of spontaneous resolution of symptoms, which should require randomized prospective studies to eliminate possible confounding variables [21]. An- other limitation is that due to the continuation of medication in some patients, there was a possibility that pharmacotherapy might have affected the outcome of IF-ES. However, an objective analysis was not possible in our study.

Although several studies have reported the safety and efficacy of IF-ES for LUTS $[6,12,14,22]$ and enuresis $[8,9]$ in children, IF-ES is not yet practiced in Korea. The reasons appear to be the limited knowledge of the modality, insufficient trained manpower for this outpatient procedure, and cost problems. Another potential limitation of ES is that long-term follow-up data are not available, and Hoebeke et al. [23] reported a high failure rate of $44 \% 1$ year after cessation of ES. Kang et al. [24] reported that because the neural development of children is immature, IF-ES treatment could achieve neuromodulation that could lead to improvement of LUTS. Considering these results, long-term studies are necessary in the future.

Based on the results of this pilot study, we are planning a prospective study with a larger number of patients and longer follow-up to investigate the efficacy and safety of IF-ES in patients with NE. Based on our research, we may provide valuable information about IF-ES, which may help establish guidelines for salvage therapy and aid clinical practice in the future.

\section{CONFLICT OF INTEREST}

No potential conflict of interest relevant to this article was reported.

\section{ACKNOWLEDGEMENTS}

We are obliged to Mrs. Yeong Ju Ma and Ms. Jinjoo Kim for their valuable help in data acquisition.

\section{REFERENCES}

1. Elmissiry M, Abdelkarim A, Badawy H, Elsalmy S, Ali GA. Refractory enuresis in children and adolescents: how can urodynamics affect management and what is the optimum test? J Pediatr Urol 2013;9:348-52.

2. Moffatt ME. Nocturnal enuresis: psychologic implications of treatment and nontreatment. J Pediatr 1989;114(4 Pt 2):697-704.

3. Lee SD, Sohn DW, Lee JZ, Park NC, Chung MK. An epidemiological study of enuresis in Korean children. BJU Int 2000;85:869-73.

4. Neveus T, Eggert P, Evans J, Macedo A, Rittig S, Tekgul S, et al. Evaluation of and treatment for monosymptomatic enuresis: a 
standardization document from the International Children's Continence Society. J Urol 2010;183:441-7.

5. Franco I, von Gontard A, De Gennaro M; International Childrens's Continence Society. Evaluation and treatment of nonmonosymptomatic nocturnal enuresis: a standardization document from the International Children's Continence Society. J Pediatr Urol 2013;9: 234-43.

6. Barroso U Jr, Tourinho R, Lordelo P, Hoebeke P, Chase J. Electrical stimulation for lower urinary tract dysfunction in children: a systematic review of the literature. Neurourol Urodyn 2011;30:1429-36.

7. Dasgupta R, Critchley HD, Dolan RJ, Fowler CJ. Changes in brain activity following sacral neuromodulation for urinary retention. J Urol 2005;174:2268-72.

8. Lordêlo P, Benevides I, Kerner EG, Teles A, Lordelo M, Barroso U Jr. Treatment of non-monosymptomatic nocturnal enuresis by transcutaneous parasacral electrical nerve stimulation. J Pediatr Urol 2010;6:486-9.

9. de Oliveira LF, de Oliveira DM, da Silva de Paula LI, de Figueiredo AA, de Bessa J Jr, de Sa CA, et al. Transcutaneous parasacral electrical neural stimulation in children with primary monosymptomatic enuresis: a prospective randomized clinical trial. J Urol 2013; 190:1359-63.

10. Emmerson C. A preliminary study of the effect of interferential therapy on detrusor instability in patients with multiple sclerosis. Aust J Physiother 1987;33:64-5.

11. Chase J, Robertson VJ, Southwell B, Hutson J, Gibb S. Pilot study using transcutaneous electrical stimulation (interferential current) to treat chronic treatment-resistant constipation and soiling in children. J Gastroenterol Hepatol 2005;20:1054-61.

12. Kajbafzadeh AM, Sharifi-Rad L, Baradaran N, Nejat F. Effect of pelvic floor interferential electrostimulation on urodynamic parameters and incontinency of children with myelomeningocele and detrusor overactivity. Urology 2009;74:324-9.

13. Kajbafzadeh AM, Sharifi-Rad L, Nejat F, Kajbafzadeh M, Talaei HR. Transcutaneous interferential electrical stimulation for management of neurogenic bowel dysfunction in children with myelomeningocele. Int J Colorectal Dis 2012;27:453-8.
14. Kajbafzadeh AM, Sharifi-Rad L, Dianat S. Efficacy of transcutaneous functional electrical stimulation on urinary incontinence in myelomeningocele: results of a pilot study. Int Braz J Urol 2010;36: 614-20.

15. Lindström S, Fall M, Carlsson CA, Erlandson BE. The neurophysiological basis of bladder inhibition in response to intravaginal electrical stimulation. J Urol 1983;129:405-10.

16. Satter EK. Third-degree burns incurred as a result of interferential current therapy. Am J Dermatopathol 2008;30:281-3.

17. Queralto M, Vitton V, Bouvier M, Abysique A, Portier G. Interferential therapy: a new treatment for slow transit constipation. a pilot study in adults. Colorectal Dis 2013;15:e35-9.

18. Lordelo P, Teles A, Veiga ML, Correia LC, Barroso U Jr. Transcutaneous electrical nerve stimulation in children with overactive bladder: a randomized clinical trial. J Urol 2010;184:683-9.

19. Ford KS, Shrader MW, Smith J, McLean TJ, Dahm DL. Full-thickness burn formation after the use of electrical stimulation for rehabilitation of unicompartmental knee arthroplasty. J Arthroplasty 2005;20:950-3.

20. Petrofsky J, Laymon M, Prowse M, Gunda S, Batt J. The transfer of current through skin and muscle during electrical stimulation with sine, square, Russian and interferential waveforms. J Med Eng Technol 2009;33:170-81.

21. Malm-Buatsi E, Nepple KG, Boyt MA, Austin JC, Cooper CS. Efficacy of transcutaneous electrical nerve stimulation in children with overactive bladder refractory to pharmacotherapy. Urology 2007;70: 980-3.

22. Oh-Oka H, Fujisawa M. Efficacy on interferential low frequency therapy for elderly overactive bladder patients with urinary incontinence. Nihon Hinyokika Gakkai Zasshi 2007;98:547-51.

23. Hoebeke P, Renson C, Petillon L, Vande Walle J, De Paepe H. Percutaneous electrical nerve stimulation in children with therapy resistant nonneuropathic bladder sphincter dysfunction: a pilot study. J Urol 2002;168:2605-7.

24. Kang SH, Bae JH, Shim KS, Park HS, Cheon J, Lee JG, et al. Extracorporeal magnetic innervation therapy in children with refractory monosymptomatic nocturnal enuresis. Urology 2007;70:576-80. 